\title{
Experimental and Computational Analysis of Residual Stress and Mechanical Hardening in Welded High-Alloy Steels
}

\author{
Nico Hempel ${ }^{1, a,{ }^{*},}$ Thomas Nitschke-Pagel ${ }^{1, b}$, Joana Rebelo-Kornmeier ${ }^{2, c}$ and \\ Klaus Dilger ${ }^{1, d}$ \\ ${ }^{1}$ TU Braunschweig, Institute of Joining and Welding, Langer Kamp 8, D-38106 Braunschweig, \\ Germany \\ ${ }^{2}$ Heinz Maier-Leibnitz Zentrum (MLZ), Technische Universität München, Lichtenbergstr. 1, D- \\ 85748 Garching, Germany \\ an.hempel@tu-braunschweig.de, bt.pagel@tu-braunschweig.de, \\ cJoana.Kornmeier@frm2.tum.de, dk.dilger@tu-braunschweig.de
}

Keywords: Welding, Residual Stress, Mechanical Hardening, Austenitic Steel, Neutron Diffraction, X-Ray Diffraction, Numerical Welding Simulation

\begin{abstract}
Due to the thermal cycle during welding, plastic deformation can occur in the heataffected zone. After cooling, the yield stress can be locally increased due to the hardening effect and thus permits higher residual stresses in these areas. Therefore, a precise description of the hardening behavior in welding simulations is indispensable for reliably predicting residual stresses. Thus, this work is dedicated to the characterization of mechanical hardening in welded high-alloy steels and its effects on the residual stress state. Numerical welding simulations are performed using different hardening models and the outcomes in terms of both the residual stress and the hardening state are compared to experimental results gained by laboratory X-ray and neutron diffraction.
\end{abstract}

\section{Introduction}

Welding simulation is a powerful tool for predicting the residual stresses and distortion due to a welding process. However, the numerical outcome strongly depends on the choice of the hardening model and can differ significantly from experimental results [1]. Since the thermomechanical welding cycle induces plastic deformation and the residual stresses can be as high as the local yield stress, modeling the cyclic hardening behavior correctly is indispensable for predicting the residual stress state, which eventually may affect the structural performance of a welded component. Hardening models used for welding simulation usually take isotropic hardening, accounting for a successive growth of the yield surface, or kinematic hardening, represented by a translation of the yield surface, or both mechanisms into account. Numerous researchers have investigated the effect of the choice of the hardening model and were able to attain good agreement with experimental results, especially when sophisticated models like the Lemaitre-Chaboche model [2] are used, see e.g. [3] for an austenitic steel.

In this paper, the first results of an ongoing research project are shown which is aimed at clarifying which hardening model should be used for the welding simulation of different steels and which modeling effort is required. To this end, a bead-on-plate dummy weld without filler material is considered both experimentally and numerically. The welding residual stresses computed with different hardening models are compared to results of diffraction experiments. Additionally, characteristic quantities of hardening derived from the simulations are compared to the microstrain, which is a microstructural indicator of material hardening. Thus, this work will eventually help to make mechanically and microstructurally based recommendations for the choice of the hardening models in welding simulations. 


\section{Experimental work}

Sample preparation. Plates of the austenitic steel X2CrNi18-9 (AISI 304L) were used for the experiments. Tensile tests at room temperature revealed a yield stress of $255 \mathrm{MPa}$ at $0.01 \%$ plastic strain. The material was heat-treated at $1050{ }^{\circ} \mathrm{C}$ for 12 minutes and cooled in air. Beadon-plate welding was then performed on workpieces of dimensions $150 \times 200 \times 10 \mathrm{~mm}^{3}$ using a mechanized tungsten inert gas (TIG) process without filler metal in flat position with a nominal heat input of $8.2 \mathrm{~kJ} / \mathrm{cm}$. The plates were placed on three ceramic balls during welding, guaranteeing accurately defined boundary conditions for the welding simulations, see also Fig. 1. Transient temperatures were recorded by NiCr-Ni thermocouples positioned at different distances from the weld.

Analysis of residual stress. The residual stresses and the mechanical hardening state in the welded samples were determined using X-ray diffraction (XRD) and neutron diffraction (ND). The measurements were taken at points along a line perpendicular to the welding direction at mid-length of the weld, see the measurement locations in Fig. 1. The residual stresses on the surface of the plates were determined by XRD using an $\Omega$-diffractometer and a cylindrical collimator with a diameter of $2 \mathrm{~mm}$. Interference lines of $\mathrm{Cr}-\mathrm{K}_{\alpha}$ radiation originating from the $\{220\}$ lattice planes of austenite were recorded in a $2 \theta$ interval of about $121-137^{\circ}$ using a $1 \mathrm{D}$ position sensitive detector. The sample was tilted for eleven angles $\psi$ of $0^{\circ}, 13^{\circ}, 18^{\circ}, 24^{\circ}, 27^{\circ}$, $30^{\circ}, 33^{\circ}, 36^{\circ}, 39^{\circ}, 42^{\circ}$ and $45^{\circ}$, allowing for an analysis of the shift of the interference line's center of gravity by the $\sin ^{2} \psi$ method. Previously, the $K_{\alpha 2}$ doublet was eliminated using the Rachinger technique and the remaining $\mathrm{K}_{\alpha 1}$ peak was smoothed with a Savitzky-Golay filter. ND measurements for residual stress analysis inside the plate were carried out at the STRESS-SPEC instrument [4] at the Heinz Maier-Leibnitz Zentrum (MLZ), Garching, Germany. The diffraction of neutrons with a wavelength of $\lambda \approx 1.67 \AA$ on the $\{311\}$ lattice planes of austenite was analyzed. Strain mapping in transverse, longitudinal and normal directions was performed using a gauge volume of $1 \times 1 \times 1 \mathrm{~mm}^{3}$ at ten depth positions and 19 different distances from the weld centerline (WCL) in order to compute the multiaxial residual stress state. The stress-free lattice spacing was determined in a cylindrical sample of diameter $3 \mathrm{~mm}$ containing both molten and base material. Diffraction elastic constants $E^{\{220\}}=256200 \mathrm{~N} / \mathrm{mm}^{2}, v^{\{220\}}=0.304, \mathrm{E}^{\{311\}}=$ $186900 \mathrm{~N} / \mathrm{mm}^{2}$ and $v^{\{311\}}=0.325$ have been determined experimentally and were used for all analyses.

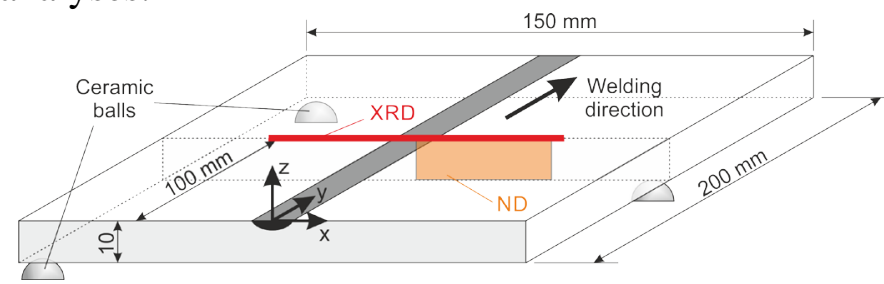

Figure 1: Experimental setup and locations of diffraction measurements

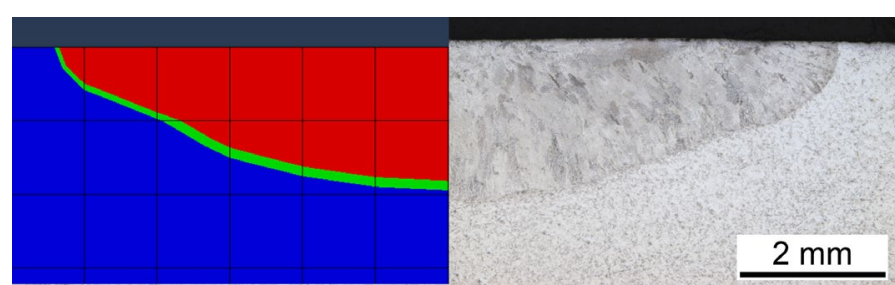

Figure 2: Simulated temperature distribution in cross-section (left) compared to macrograph (right) with $T>T_{\text {liquidus }}$ (red) and $T>T_{\text {solidus }}$ (green)

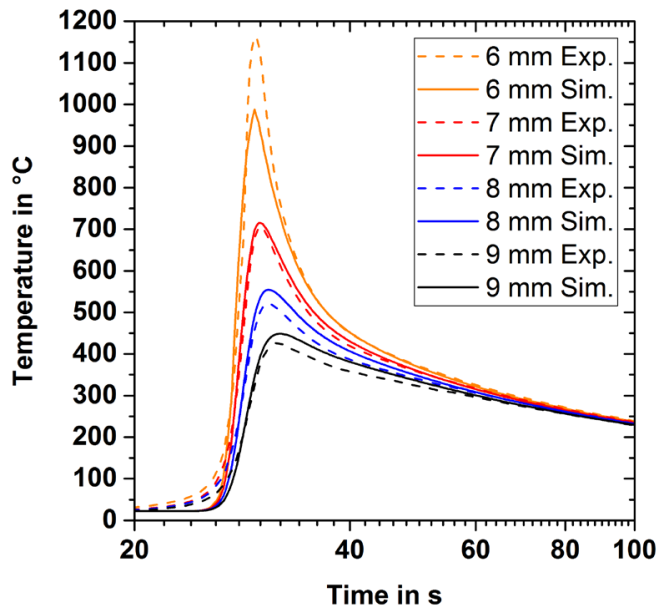

Figure 3: Transient temperatures at different distances from the WCL; experiment and simulation

Analysis of mechanical hardening. Mechanical hardening usually leads to an increase of the dislocation density and thus microstrain within the atomic lattice of a polycrystal. Therefore, the 
microstrain is an indicator of mechanical hardening, which can be determined using diffraction line profile analysis. To this end, the diffraction lines recorded in the normal direction of a welded sample as well as $\mathrm{LaB}_{6}$ powder standard samples were utilized for both XRD and ND. Instrumental broadening was eliminated by Fourier deconvolution [5] and the remaining physical profiles were fitted using a pseudo-Voigt function. From its width parameters the microstrain was computed [6].

\section{Numerical simulations}

Welding simulations with the finite element method (FEM) were carried out using the software Abaqus. The FE mesh consisted of 37760 linear hexahedral elements with 43170 nodes and had a size of $1 \times 2.5 \times 1 \mathrm{~mm}^{3}$ in the weld and heat-affected zone. The thermal and mechanical boundary conditions of the welding experiments were replicated in the simulation. First, a thermal simulation was run using a circular surface heat source with constant distribution, whose parameters were fit to a macrograph, see Fig. 2, and temperature measurements, see Fig. 3, for an optimal representation of the actual TIG process. The transient temperatures were then input into the mechanical simulation where the residual stresses were computed. The temperature dependent physical properties were taken from a literature study by Voß [7] for the similar steel X5CrNi18-10. Since temperature-dependent stress-strain are not yet available for the X2CrNi189 steel investigated here, quasi-static flow curve data has also been taken from [7] and was scaled by fitting the flow curve at room temperature used by Voß to the one determined by tensile tests of X2CrNi18-9 at room temperature. In order to study the effect of the hardening model, the simulations were carried out with isotropic, kinematic and ideally plastic material behavior. For all cases, the combined isotropic-kinematic hardening law in Abaqus [2] was used, setting kinematic, isotropic or both hardening mechanisms to zero, respectively. For isotropic and kinematic hardening, the flow curves were modeled by the same asymptotic exponential functions, guaranteeing that the hardening behavior is quantitatively identical and differs only qualitatively. The annealing temperature, where the strain history is reset, is assumed to be 1000 ${ }^{\circ} \mathrm{C}$, which is close to the solution heat treatment temperature of austenitic steels. Simulations with actual material data based on tensile and cyclic tests performed on X2CrNi18-9 will be shown in a later study.

\section{Experimental results}

Fig. 4 shows the longitudinal, transverse and von Mises residual stresses on the surface. It can be seen that the longitudinal residual stress component takes low compressive values far away from the weld, enters the tensile regime at about $20 \mathrm{~mm}$ from the WCL and reaches a maximum of about $550 \mathrm{MPa}$ at about $8 \mathrm{~mm}$ from the WCL. The residual stress then decreases to about 300 $\mathrm{MPa}$ at the weld toe. Due to the coarse grain in the molten zone, the XRD measurements could not be analyzed there. The von Mises stress largely follows the longitudinal stress component, showing a maximum of about $500 \mathrm{MPa}$ at about $8 \mathrm{~mm}$ from the WCL. In contrast to the longitudinal and von Mises residual stresses, the transverse component is not fully symmetric with respect to the WCL, which might be due to the fact that only tilt angles $\psi>0^{\circ}$ have been used and the negative side of the plate was sloped because of the welding distortion. Considering only the results on the positive side of the WCL, the transverse residual stress exhibits a maximum of about $240 \mathrm{MPa}$ at $12 \mathrm{~mm}$ from the WCL and lower tensile values at the other positions. In general, it can be observed that the measurement uncertainty, resulting from the linear $\sin ^{2} \psi$ fit, is rather high close to the weld toe, which is especially true for the transverse residual stress.

Due to space constraints, only the von Mises equivalent residual stress determined from the ND measurements is shown here, see Fig. 5. The highest equivalent residual stresses of up to 350 MPa are found around the molten zone that is indicated by the dashed line in the top left corner. While the band of the highest equivalent residual stresses is rather narrow near the top surface at 
about $8 \mathrm{~mm}$ from the WCL, it is wider below the molten zone and extends to the bottom of the plate.

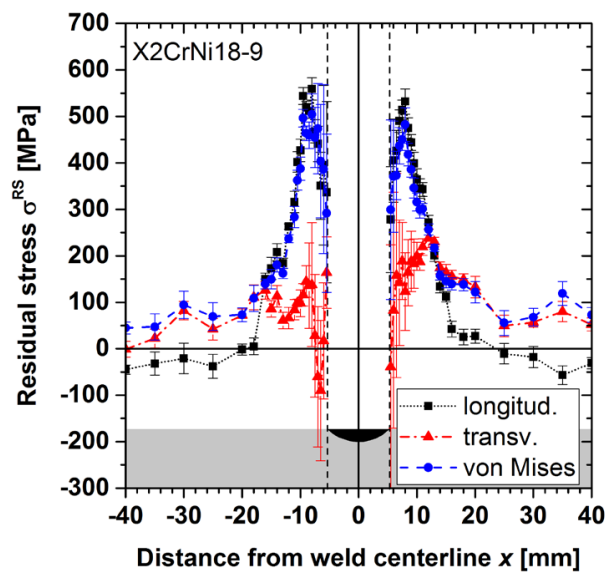

Figure 4: Longitudinal, transverse and von Mises residual stress on the plate surface

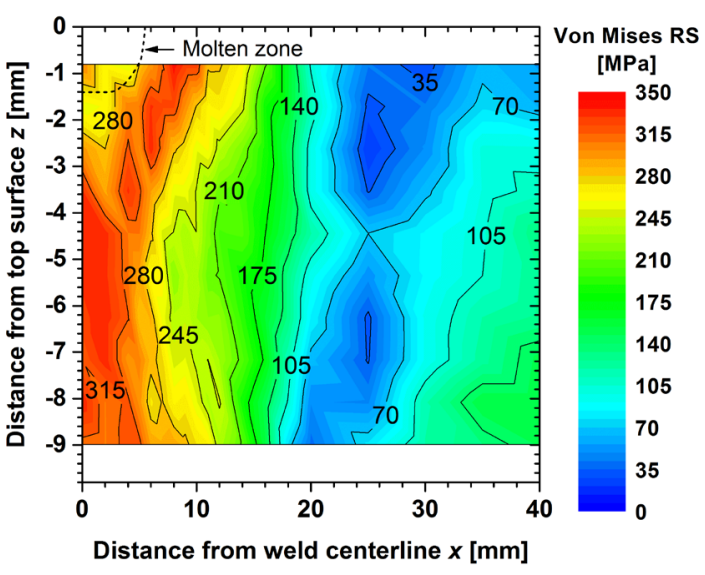

Figure 5: Von Mises residual stress in the cross-section of a welded plate

In Fig. 6 the microstrain as determined by line profile analysis of the XRD measurements is shown along a line on the surface of the plate. The distribution is almost symmetric with respect to the WCL, except for two outliers, and takes maximum values at $8 \mathrm{~mm}$ from the WCL. High gradients can be observed to both sides of the maxima. The microstrain takes relatively constant low values at distances larger than about $20 \mathrm{~mm}$ from the WCL.

The microstrain distribution derived from the ND measurements is depicted in Fig. 7. It can be seen that the molten zone is surrounded by an area with values that are higher than in the base material far away from the WCL. The maximum is found near the surface at about $7 \mathrm{~mm}$ from the WCL. It can also be observed that the values are of a similar magnitude than the results of the XRD measurements, see Fig. 6, but the relative difference between the minimum and maximum values is much lower for the ND results.

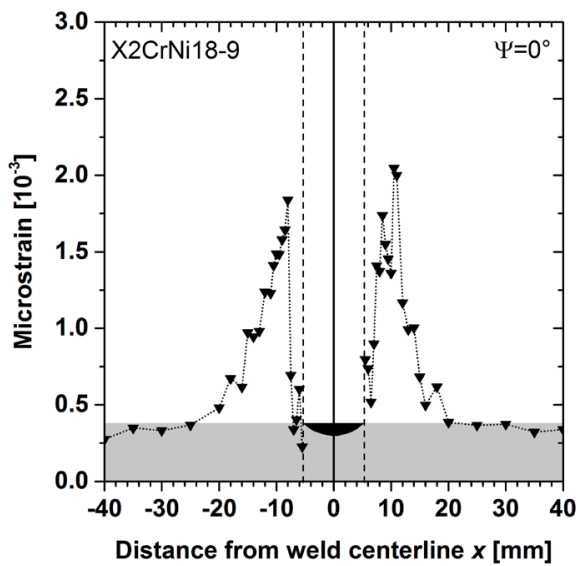

Figure 6: Microstrain distribution on the surface of a welded plate

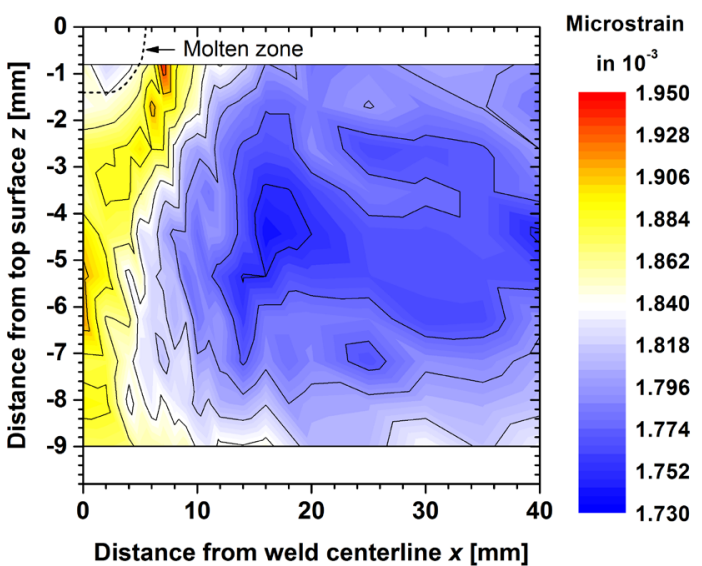

Figure 7: Microstrain distribution in the crosssection of a welded plate

\section{Numerical results}

A comparison of the numerical results obtained with different hardening models and the XRD measurements in terms of the von Mises equivalent residual stress is presented in Fig. 8. It can be seen that the three different hardening models yield qualitatively distinctly different results, especially within $20 \mathrm{~mm}$ of the WCL. Here, the isotropic model yields the highest residual stresses, taking maxima of $420 \mathrm{MPa}$ at $7 \mathrm{~mm}$ from the WCL and showing slightly lower values in the molten zone, thus being closer to the experimental results than the other simulations. The 
results of the kinematic model exhibit a sharp bend at $20 \mathrm{~mm}$ from the WCL, remain on a level of about $200 \mathrm{MPa}$ and increase to $330 \mathrm{MPa}$ in the molten zone. The results of the ideally plastic model show the same bend, but remain at a constant plateau of $207 \mathrm{MPa}$.

In order to highlight the effect of the hardening model, the characteristic quantities for isotropic and kinematic hardening, i.e. the change of size of the isotropic yield surface $\Delta \sigma_{\mathrm{y}}$ and the absolute value of the kinematic yield surface translation tensor $\|\boldsymbol{\alpha}\|$ after cooling are presented in Fig. 9. It can be seen that isotropic hardening leads to a yield stress increase of up to $220 \mathrm{MPa}$ in the heat-affected zone, whereas the values in the molten zone are about $130 \mathrm{MPa}$. Kinematic hardening, however, yields only a small residual effect on the yield surface in the heat-affected zone, but a much more pronounced effect in the molten zone, showing translations of the yield surface of about $12 \mathrm{MPa}$ and $130 \mathrm{MPa}$, respectively.

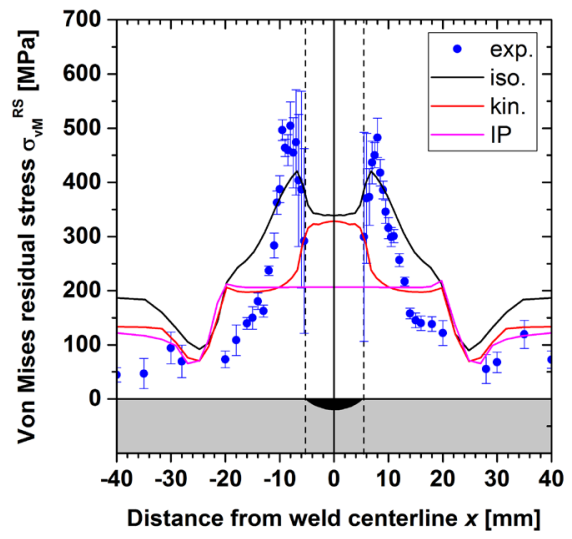

Figure 8: Von Mises residual stress determined by XRD (exp.) and computed with isotropic (iso.), kinematic (kin.) and no (IP) hardening

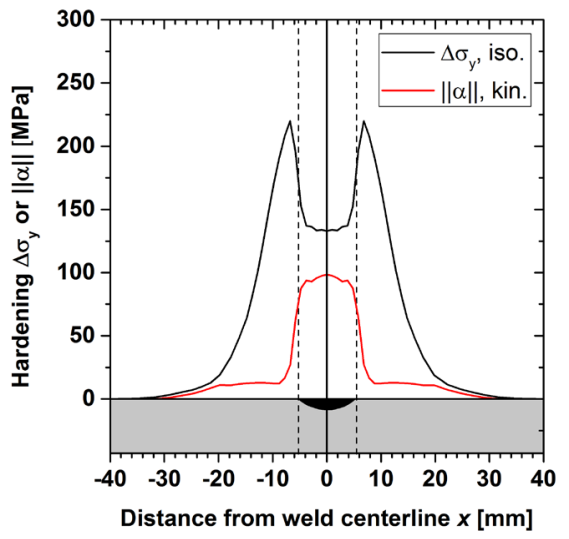

Figure 9: Change of size of isotropic yield surface $\Delta \sigma_{y}$ and absolute value of kinematic yield surface translation tensor $\|\alpha\|$

\section{Discussion}

Diffraction measurements revealed equivalent residual stresses that significantly exceed the initial yield stress of $255 \mathrm{MPa}$, see Figs. 4 and 5. For the welding geometry investigated here, the longitudinal stress component is dominant because of the self-constraint imposed by the plate, whereas the transverse residual stresses are relatively low due to free angular shrinkage.

The distributions of the microstrain, see Figs. 6 and 7, indicate significant mechanical hardening that must have occurred locally due to the thermomechanical welding cycle, permitting higher residual stresses. This is proven by the fact that the microstrain distributions coincide well with the areas with the highest residual stresses shown in Figs. 4 and 5.

The pronounced hardening effect explains why different hardening models yield distinctly different numerical results within $20 \mathrm{~mm}$ from the WCL. Here, the isotropic model gives an equivalent residual stress distribution that is qualitatively equal to the experimental one, see Fig. 8. Differences in the maximum residual stresses and the wider computed residual stress profile can be explained by the fact that external material data has been used which was only fitted to test results at room temperature. This cannot replace a full material characterization which is currently under progress and whose results will be used in future work.

The simulations with the kinematic model, however, underestimate the residual stresses in the heat-affected zone significantly. This is due to the fact that only a translation of the yield surface is considered. During heating, compressive stresses develop in the heat-affected zone due to the constrained thermal expansion, leading to plastic deformation and thus a translation of the yield surface in a certain direction. During cooling, however, the constrained shrinkage of the highlyheated areas results in tensile stresses and thus an earlier onset of plastic yield due to the previous shift of the yield surface. Thereby, the yield surface is shifted in almost opposite 
direction than before. In fact, this re-translation is not exactly opposite due to the nonproportional multiaxial stress state, but nevertheless leads to a rather small residual translation in the heat-affected zone, see $\|\boldsymbol{\alpha}\|$ in Fig. 9.

Comparing the computational hardening effects, shown in Fig. 9, to the microstrain, which is an indicator of dislocations and thus mechanical hardening, it can be seen that only the results derived from the isotropic model are in qualitative agreement with the experimental findings, see Fig. 6. Thus, it can be inferred that the isotropic hardening model not only yields qualitatively correct residual stress distributions, but its choice is also legitimated by the microstructural mechanisms caused by the thermomechanical welding cycle.

\section{Summary and future work}

In this work, the effect of the choice of the hardening model in numerical welding simulations was analyzed studying a bead-on-plate weld on austenitic steel. It was found that the isotropic hardening model yields qualitatively correct residual stress distributions and that the continuum mechanical hardening effect agrees well with microstructural findings. This was neither the case for the kinematic hardening model nor for ideal plasticity.

However, these investigations could only yield qualitative results regarding the numerical simulations since material data from the literature has been used. Future work will therefore comprise the use of real material data, which also allows for the use of more sophisticated models of cyclic plasticity. Furthermore, the analysis of multilayer welds, i.e. of the effect of several thermomechanical cycles, and further microstructural analyses are planned.

\section{Acknowledgement}

This work was carried out in the research project Ni508/14-1 which was generously supported by the Deutsche Forschungsgemeinschaft (DFG). The authors would like to express their thanks for the support.

This work is based upon experiments performed at the Stress-Spec instrument operated by Helmholtz-Zentrum Geesthacht (HZG) at the Heinz Maier-Leibnitz Zentrum (MLZ), Garching, Germany. The authors gratefully acknowledge the financial support provided by HZG to perform the neutron scattering measurements at the MLZ.

\section{References}

[1] H. Wohlfahrt, Th. Nitschke-Pagel, K. Dilger, D. Siegele, M. Brand, J. Sakkiettibutra, T. Loose, Residual stress calculations and measurements - review and assessment of the IIW round robin results, Weld. World 56 (2012) 09/10, 120-140.

[2] J. Lemaitre, J.L. Chaboche, Mechanics of solid materials, Cambridge University Press, 1990.

[3] M.C. Smith, O. Muránsky, C. Austin, P. Bendeich, Q. Xiong, Optimised modelling of AISI 316L(N) material behaviour in the NeT TG4 international weld simulation and measurement benchmark, Int. J. Pres. Ves. Pip. (in press), https://doi.org/10.1016/j.ijpvp.2017.11.004.

[4] Heinz Maier-Leibnitz Zentrum, STRESS-SPEC: Materials science diffractometer, Journal of large-scale research facilities, 1, A6 (2015). http://dx.doi.org/10.17815/jlsrf-1-25.

[5] A.R. Stokes, A numerical Fourier-analysis method for the correction of widths and shapes of lines on X-ray powder photographs. Proc. Phys. Soc. 61 (1948) 382-391.

[6] T.H. de Keijser, J.I. Langford, E.J. Mittemeijer, A.B.P. Vogels, Use of the Voigt function in a single-line method for the analysis of X-ray diffraction line broadening, J. Appl. Cryst. 15 (1982) 308-314.

[7] O. Voß, Untersuchung relevanter Einflussgrößen auf die numerische Schweißsimulation, Dissertation, TU Braunschweig, 2001 (in German). 